\title{
HIV seroprevalence and factors affecting clinical outcomes among patients with surgical acute abdomen in selected hospitals in Mwanza, north- western in Tanzania
}

\author{
PRAVEEN SRAVANAM ${ }^{1}$, ALICIA MASSENGA ${ }^{1,3}$, MERCHADES BUGIMBI $^{1,2}$, MARIAM MIRAMBO 4 and PHILLIPO \\ L. CHALYA ${ }^{2^{*}}$ \\ ${ }^{1}$ Department of Surgery, Catholic University of Health and Allied Sciences-Bugando, Mwanza, Tanzania \\ 2Department of Surgery, Bugando Medical Centre, Mwanza, Tanzania \\ 3Department of Paediatric Surgery, Bugando Medical Centre, Mwanza, Tanzania \\ ${ }^{4}$ Department of Microbiology and Immunology, Catholic University of Health and Allied Sciences-Bugando, \\ Mwanza, Tanzania
}

\begin{abstract}
Background: HIV infection has been reported to be prevalent in patients with surgical acute abdomen. This study aimed to establish HIV seroprevalence among patients with surgical acute abdomen and to identify factors affecting clinical outcomes among these patients at a tertiary care hospital in Tanzania

Methods: This was a prospective cross-sectional study of patients with surgical acute abdomen admitted to three selected hospitals in Mwanza between February 2016 and July 2016.

Results: Out of the 106 patients (M: $F=1.5: 1)$ included in the study, 15 (14.2\%) were HIV positive. Of these, nine were males and 6 were females. Surgical site infection was the most common postoperative compilation and was found to be significantly higher in HIV positive patients with CD 4+ count below 200 cells/ $\mu$ l ( $p<0.001)$. The median length of hospital stay (LOS) was 16 days. There was no significant difference in LOS between HIV positive and negative patients $(p=0.791)$. Mortality rate was $13.2 \%$ and it was significantly higher in patients with advanced age (> 65 years), pre-existing medical illness, delayed presentation ( $>48$ hours), HIV positivity, high American Society of Anesthetists class, surgical site infections $(p<0.001)$.

Conclusion: This study has shown that HIV infection is prevalent among patients with surgical acute abdomen in our setting. Factors influencing the clinical outcome of these patients need to be addressed, in order to deliver optimal patient care and improve their treatment outcome.
\end{abstract}

Keywords: HIV, seroprevalence, clinical outcome, surgical acute abdomen, Tanzania

\section{Introduction}

Surgical acute abdomen remains one of the most common and potentially dangerous and lifethreatening surgical emergency with high morbidity and mortality worldwide (Morales et. al. 2017). Globally, an estimated 5-10\% of all emergency department visits is due to surgical acute abdomen (Bhuiya et. al. 2010; Hastings \& Powers, 2011; Khojah et. al. 2017; Morales et. al. 2017). In Tanzania, surgical acute abdomen continue to be one of the leading causes of morbidity and mortality and at Bugando Medical Centre and other hospitals in Mwanza region, it is the single commonest indication for admission reported in the surgical wards (Chalya et al. 2011, 2013a,b; Mabula et al. 2012; Mabewa et al, 2015).

The management of surgical acute abdomen poses a diagnostic and therapeutic challenges especially in developing countries like Tanzania where HIV-related disease continues to increase and presents the greatest single challenge to the medical practitioners seeking to alleviate suffering (Mabula et al. 2012; Chalya et al. 2013a; Mabewa et al, 2015). Surgical acute abdomen in the HIV-AIDS patients involves abnormal presentation of common diseases as well as problems unique to this population, which are results of the immunosuppression (Aboolian et. al. 1999; Owotade et.al. 2003). HIV infection complicates the care of patients with surgical acute abdomen

\footnotetext{
*Correspondence: E-mail plchalya65@gmail.com
} 
and is associated with increased postoperative complications in these patients (Amita et al., 2008; Amira \& Awobusuyi, 2014).

The HIV seroprevalence among patients with surgical acute abdomen is currently not known in our setting. Establishing the seroprevalence of HIV among patients with surgical acute abdomen is of paramount importance for education and post-exposure prophylaxis. Previous studies done at Bugando Medical Centre have indicated that the outcome of patients with surgical acute abdomen has remained poor (Mabula et al. 2012; Chalya et al., 2013a; Mabewa et al., 2015). The reasons for this state of affairs have not been evaluated. HIV infection has been reported to have an effect on the clinical outcomes of patients with surgical acute abdomen (Gazard et al., 1999; Davis et al., 2001; Dua \& McWinlet, 2007). This observation has also not been evaluated in our environment. This study aimed to establish the seroprevalence of HIV infection among patients with surgical acute abdomen and to identify factors affecting their clinical outcomes at selected hospitals in north-western Tanzania.

\section{Materials and Methods}

\section{Study design and setting}

This was a prospective cross sectional study which was conducted at the Accident and Emergency department of Bugando Medical Centre (BMC), Sekou-Toure Region Referral hospital (STRRH) and Sengerema Designated District Hospital (SDDH) during February 2016 to July 2016. These three health facilities were conveniently selected because of their close proximity in the north-western Tanzania. BMC is a referral, consultant, tertiary care and teaching hospital for the Catholic University of Health and Allied Sciences-Bugando (CUHAS-Bugando) and other paramedics and it is located in Mwanza city. It is situated along the shore of Lake Victoria and has 890 beds. BMC is one of the four largest referral hospitals in the country and serves as a referral centre for tertiary specialist care for a catchment population of approximately 13 million people from neighbouring regions. On average on a day 4-6 patients are treated with surgical acute abdomen at BMC. STRRH is a public regional facility serving the population of Mwanza Region on the shore of Lake Victoria in north-western Tanzania. It is a 450-bed hospital that serves a catchment area with a population of 1.3 million people. SDDH is a designated district hospital in Mwanza region and has a bed capacity of 330 and a catchment population of 772,581. The hospital serves a catchment area of half a million people while simultaneously serving as a training site for undergraduate medical students, nursing students and low-level medical providers.

\section{Study population}

The study population included all patients presenting with surgical acute abdomen admitted and treated at the three hospitals and who consented for the study and HIV testing. Recruitment of patients was done at the emergency department and in the surgical wards after initial resuscitation was done by the admitting surgical team. After informed consent to participate in the study and to be tested for HIV, all patients who met the inclusion criteria were consecutively enrolled into the study. All patients were pre-counselled by a professional HIV counsellor. The patients were offered to be informed of the result of the HIV test. When personal consent was not possible due to the nature of the disease or age below 18 years, consent was sought from the family. Patients were assured that the result would not affect their management and were given the choice to know their results. To prevent bias the medical and nursing staff were not informed about the results of the HIV test. All patients were treated according to the current protocol in respective hospitals which, given the very high prevalence of HIV in general population, assumes that all patients are potentially infected with HIV and staff anticipate HIV related complications and problems in nursing care as a part of daily routine.

On admission, a sample of blood was taken from the patients and used to carry out HIV serology test, CD4+cell count and complete blood count. HIV serology test was performed using 
the Tanzania HIV Rapid Test Algorithm (Lyamuya et al, 2009). CD4+ cell count was performed using FACS Count or FACSCALIBUR (BD Biosciences USA), to all HIV positive patients to determine the degree of immunosuppression. Imaging studies like $x$-rays, ultrasound and Computer Tomography (CT) scan were also taken depending on the type of injury.

All recruited patients were managed according to the current protocol in respective hospitals. Patients were followed up until discharge or death. The length of hospital stay (LOS) and mortality as measures of outcome of trauma patients were recorded at the end of follow up period. LOS was categorized as less than 14 days and more than 14 days as prolonged hospital stay. HIV positive patients were referred to the Care and Treatment Clinic (CTC) after post- counselling.

\section{Data collection}

Data was collected using a pre-tested coded questionnaire. Information sought included demographic characteristics, cause of acute abdomen, duration of symptoms, clinical presentation, HIV status, radiological findings, timing of surgical procedure, American Society of Anesthetists (ASA) classification, operative findings and surgical procedure performed. Outcome was measured in terms of postoperative complications, length of hospital stay and mortality.

\section{Data analysis}

Data analysis was performed using Statistical Package for Social Sciences (SPSS) version 20.0 for Windows (SPSS, Chicago IL, USA). The median and Interquartile Range (IQR) and ranges were calculated for continuous variables whereas proportions and frequency tables were used to summarize categorical variables. Chi-square $\left(\chi^{2}\right)$ test was used to test for the significance of association between the independent (predictor) and dependent (outcome) variables in the categorical variables. The level of significance was considered as $p<0.05$. Study variable that was found to be statistically significant in univariate analysis were subjected to multivariate logistic regression analysis. Multivariate logistic regression analysis was used to determine predictor variables that predict the postoperative complications, hospital stay and mortality.

\section{Ethical consideration}

The ethical approval to conduct the study was sought from CUHAS/BMC Research Ethics and Review Committee (CREC/125/2016). The permission to conduct the study was sought from respective hospital authorities. Parent or guardian or patient included in the study had to sign a written informed consent before recruitment into the study. In addition an assent was obtained from children aged 5-17 years. Confidentiality was assured and the study did not interfere with the decision of the attending physician in case of absence of the investigator.

\section{Results}

\section{Patient's characteristics}

During the study period, a total of 118 patients with surgical acute abdomen were managed in the three hospitals. Of these, 12 were excluded from the study due to failure to meet the inclusion criteria. Thus, a total of 106 patients were studied. Out of these, $62(58.5 \%)$ were males and 44 (41.5\%) were females with the male to female ratio of $1.4: 1$. The ages of patients at presentation ranged from 1 day to 74 years with a mean age of $30.6 \pm 12.4$ years. The modal age group was 2130 years.

Out of the 106 patients included in the study, 15 (14.2\%) tested HIV positive and this formed the study group. The remaining 91 patients (85.8\%) formed the control group. Of the HIV positive patients, $9(60.0 \%)$ were males and $6(40.0 \%)$ were females with a male to female ratio of $1.5: 1$. This gender difference was not statistically significant $(p=0.793)$. The ages of patients at diagnosis ranged from 18 day to 56 years with a mean of $36.7 \pm 16.8$ years. The HIV-positive patients were significantly older than the HIV negative patients ( 36.7 vs. 30.2 years; $p=0.011$ ). Four $(26.7 \%)$ 
patients had stigmata of AIDS. Majority of HIV positive patients (60.0\%) had clinical stage I. Of the HIV positive patients, $4(26.7 \%)$ were known cases on highly active antiretroviral therapy (HAART) and the remaining $11(73.3 \%)$ were newly diagnosed patients. CD4+ count among HIV positive patients ranged from 68 cells $/ \mu$ lo 912 cells $/ \mu \mathrm{l}$ with the mean of $338 \pm 211$ cells $/ \mu \mathrm{l}$. Of the $15 \mathrm{HIV}$ positive patients, $10(66.7 \%)$ had $C D 4+$ count below 200 cells $/ \mu$ l and the remaining $5(33.3 \%)$ had CD4+ count of $\geq 200$ cells $/ \mu$ l. Among the HIV infected patients, $6(40.0 \%)$ reported to have preexisting medical illness namely pulmonary tuberculosis (4 patients), peripheral vascular disease with gangrene of left foot and unexplained medical jaundice in one patient each, respectively.

\section{Aetiology of surgical acute abdomen}

Table 1 below shows aetiology of surgical acute abdomen among HIV positive and HIV negative surgical patients at the three hospitals. HIV infection was found to be significantly associated with increased rate of acute appendicitis, perforated gut/viscera, intra-abdominal abscess and nonspecific abdominal pain $(\mathrm{p}<0.001)$.

Table 1: Aetiology of surgical acute abdomen among HIV positive and HIV negative surgical patients

\begin{tabular}{llll}
\hline Aetiology & HIV positive (N= 15) & HIV negative (N= 91) & p-value \\
\hline Acute intestinal obstruction & $5(33.3 \%)$ & $27(29.7 \%)$ & 0.567 \\
Acute appendicitis & $12(80.0 \%)$ & $14(15.4 \%)$ & 0.012 \\
Perforation of gut/viscera & $8(53.3 \%)$ & $7(7.7 \%)$ & 0.001 \\
Intra-abdominal abscess & $9(60.0 \%)$ & $3(3.3 \%)$ & 0.003 \\
Non-specific abdominal pain & $6(40.0 \%)$ & $4(4.4 \%)$ & 0.011 \\
Abdominal trauma & $0(0.0 \%)$ & $7(7.7 \%)$ & - \\
Biliary colic & $0(0.0 \%)$ & $3(3.3 \%)$ & - \\
Testicular torsion & $(0.0 \%)$ & $1(1.1 \%)$ & - \\
Acute cholecystis & $0(0.0 \%)$ & $1(1.1 \%)$ & - \\
\hline
\end{tabular}

\section{Treatment modalities of surgical acute abdomen}

A total of 85 (80.2\%) patients underwent surgical treatment and the remaining $21(19.8 \%)$ patients who had paralytic ileus, non-specific abdominal pain (NSAP), appendicular mass and those who had acute cholecystitis were treated conservatively (Table 2). The time interval between the onset of symptoms and definitive surgery ranged from 1-16 days with a mean of $6.45 \pm 2.32$ days.

Table 2: Operative findings among patients who underwent surgical treatment $(\mathrm{N}=85)$

\begin{tabular}{lll}
\hline Operative findings & Frequency & Percentage \\
\hline Dilated loops of bowel & 32 & 37.6 \\
Purulent peritonitis & 15 & 17.6 \\
Obstructed hernias & 11 & 12.9 \\
Hemoperitoneum & 7 & 8.2 \\
Obstructive bands and adhesions & 7 & 8.2 \\
Gangrenous bowel loops & 6 & 7.1 \\
Bowel perforations & 6 & 7.1 \\
Perforated peptic ulcers & 6 & 7.1 \\
Splenic injury & 4 & 4.7 \\
Volvulus \pm ileo-sigmoid knotting & 4 & 4.7 \\
Anorectal malformations & 3 & 3.5 \\
Testicular torsion & 2 & 2.4 \\
Mesenteric lymphadenopathy & 1 & 1.2 \\
Malignancies & 1 & 1.2 \\
Perforated gall bladder & 1 & 1.2 \\
\hline
\end{tabular}


Appendectomy was the most frequent surgical procedure performed in 20 (23.5\%) patients while tumour resection and Cholecystectomy were the least frequent at $1(1.2 \%)$ each respectively (Table 3). There was no significance difference in terms of treatment modalities, operative findings and time interval between the onset of symptoms and definitive surgery between HIV positive and negative patients $(p>0.001)$.

Table 3: Surgical procedures performed $(\mathrm{N}=85)$

\begin{tabular}{lll}
\hline Surgical procedures performed & Frequency & Percentage \\
\hline Appendectomy & 20 & 23.0 \\
Herniorrhaphy & 11 & 20.1 \\
Release of bands and adhesions & 7 & 8.2 \\
Bowel resection and anastomosis & 7 & 8.2 \\
$\begin{array}{l}\text { Repair of bowel perforation } \\
\text { Graham's omental patch (Graham's }\end{array}$ & 6 & 7.1 \\
Omentopexy) & 6 & 7.1 \\
Colostomy & 4 & \\
Exteriorization of perforation \pm ileostomy & 4 & 4.7 \\
Splenectomy & 3 & 4.7 \\
Tumour resection & 1 & 3.5 \\
Cholecystectomy & 1 & 1.2 \\
\hline
\end{tabular}

\section{Treatment outcome among HIV and non-HIV surgical patients with surgical acute abdomen}

Generally, in this study, a total of 47 post-operative complications were recorded in $28(32.9 \%)$ patients. Of these, surgical site infection was the most common post-operative complication accounting for $29.8 \%$ of cases (Figure 1 ). In the present study, the rate of surgical site infection was found to be significantly higher in HIV positive patients than in non HIV patients ( $p=0.000)$. Also, a higher rate of surgical site infections was observed among HIV patients with CD 4+ count below 200 cells/ $\mu$ l than in patients with CD $4+$ count above 200 cells $/ \mu l(p=0.002)$.

The overall length of hospital stay (LOS) ranged from 1 to 64 days with a median of 16 days (IQR $=14$ to 18 days). The median LOS for non-survivors was 3 days (range 1-16days). Patients who developed enterocutaneous fistula and those who had repeated surgery due to early postoperative complications stayed longer in the hospital and this was statistically significant ( $p$ $<0.001)$. There was no significant difference in hospital stay between HIV positive and negative patients $(p=0.791)$.

In general, fourteen patients died in this study giving a mortality rate of $13.2 \%$. Ten out of 15 (66.7\%) HIV positive patients died compared to 4 out of 91 (4.4\%) HIV negative patients which is significant $(\mathrm{p}=0.011)$. The mortality rate for HIV positive patients with stigmata of AIDS was $75.0 \%$ ( 3 out of 4 patients) compared to $18.2 \%$ ( 2 out of 11 patients) for HIV positive patients without stigmata of AIDS. These differences were statistically significant $(p=0.002)$. Mortality rate for HIV infected patients with CD4 count $<200$ cells $/ \mu \mathrm{L}$ was $70.0 \%$ (7 out of 10 patients died in this group) which is significantly higher compared to $40.0 \%$ ( 2 out of 5 patients) for HIV positive patients with $\mathrm{CD} 4$ count $\geq 200$ cells $/ \mu \mathrm{L}(\mathrm{p}=0.022)$. According to multivariate logistic regression analysis, advanced age $>65$ years [OR=4.5; 95\% $\mathrm{Cl}(2.4-8.1) ; \mathrm{p}=0.011)$, pre-existing medical illness [OR=7.3, $95 \% \mathrm{Cl}(2.4-12.3), \mathrm{p}=0.004)$, delayed presentation $>48$ hours ( $\mathrm{OR}=6.2 ; 95 \% \mathrm{Cl}(2.8-8.3) ; \mathrm{p}=0.021), \mathrm{HIV}$ positivity [OR= 2.7; 95\% (1.1-6.1), $p=0.001)$; low $C D 4$ count ( $O R=1.1 ; 95 \% \mathrm{Cl}(2.1-8.5), p=0.000)$; high ASA class $(O R=11.1 ; 95 \% \mathrm{Cl}(8.6-12.2), p=0.021)$, surgical site infections $(O R=1.2(1.0-4.6), p=0.041)$ were the main independent predictors of mortality. 


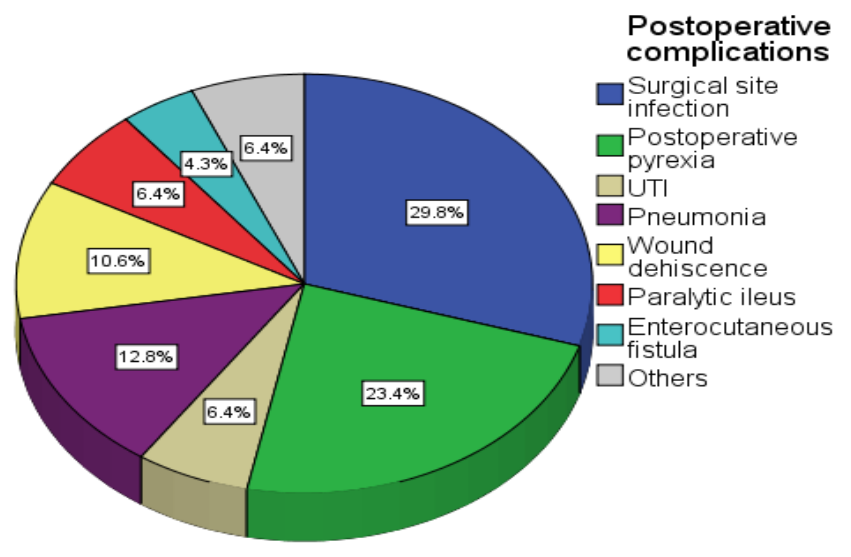

Figure 1: Distributions of patients according to postoperative complications (Key: UTI= urinary tract infection)

\section{Discussion}

The overall seroprevalence of HIV infection in our study was $14.2 \%$ that is higher than that in the general population in Tanzania (4.7\%) (Tan et. al. 2015; Johnston et. al, 2016). Similar observations were also reported by other studies (Mkony et.al. 2003; Giiti et. al. 2010; Mabula et al. 2012; Chalya et al. 2013; Mabewa et al, 2015). The prevalence of HIV infection in patients with surgical acute abdomen has been reported to be higher than in general population (Amita et al., 2008; Amira \& Awobusuyi, 2014). We could not establish the reasons for the high HIV seroprevalence in our study although it may be possible that HIV infection is a risk factor for surgical acute abdomen. However, the overall HIV seroprevalence in our study may underestimate the magnitude of the problem and may not be apparent because many cases were excluded from the study due to failure to meet the inclusion criteria and some of patients may be were still in the window period.

In agreement with other studies (Chalya et al., 2013a; Mabewa et al., 2015), HIV infection among patients with surgical acute abdomen in the present study was more common in males than in females. This finding is at variance with other studies which reported female preponderance (Giiti et al., 2010; Green et al., 2017). The exact reason for this gender difference is not known although it may reflect difference in risk factor for exposure to HIV infection that may lead to surgical acute abdomen.

As reported by other studies (Cacala et al., 2006; Makanga et al., 2006), large number of HIV infected patients were in clinical stage I (asymptomatic HIV infected patients without stigmata of HIV/AIDS) highlighting the importance of prevention and strict use of universal precautions among health care workers who care for these patients. The US Centers for Disease Control and Prevention (CDC) recommends routine screening for HIV with informed consent, for patients between 15 to 54 years of age in regions of high HIV prevalence (CDC, 2006). However, in most facilities in low-income countries, patients are not routinely screened for HIV infection and therefore little is known about the magnitude of the problem and the risk of HIV transmission among health care workers. Compliance with universal precautions has been reported to reduce the risk of HIV transmission among health care workers. However, recent studies have shown that compliance with universal barrier precautions in the high-risk setting of emergency rooms, surgical suites, and critical care units in low-income countries is less than optimal (Amita et Al., 2008; Amira 
\& Awobusuyi, 2014; Chalya et al., 2016). This observation calls for routine HIV screening all surgical patients in order to reduce the risk of HIV transmission among health care workers.

In the present study, HIV infection was found to be significantly associated with increased rate of acute appendicitis, perforated gut/viscera, intra-abdominal abscess and non-specific abdominal pain. This finding is in agreement with the report from other studies (Gazard et al., 1999; Davis et al., 2001; Dua \& McWinlet, 2007). This observation could be attributed to underlying immunosuppression in HIV seropositive patients as measured by $\mathrm{CD}_{4}^{+}$counts. HIV infection has been reported to have an impact on the outcome of patients with surgical acute abdomen (Gazard et al., 1999; Davis et al., 2001; Dua \& McWinlet, 2007). In the present study, the rate of surgical site infections and mortality was found to be significantly higher in HIV positive patients than in nonHIV patients. Also higher rate of surgical site infections was observed amongst HIV patients with low CD 4 cell count $(<200$ cells $/ \mu$ l). The reason for this observation is that HIV infection causes impaired immunity predisposing the patient to surgical wound complications such as surgical site infections. Similar observation was reported by Mawalla et al. (2011) at Bugando Medical Centre. The duration of HIV positivity and HAART use have been reported in literature to affect the clinical outcome of patients with surgical acute abdomen (Amira \& Awobusuyi, 2014; Green et al., 2017). However, in this study we could not assess the effect of these factors on the clinical outcome of these patients.

Our study found no significant difference in hospital stay between HIV positive and negative patients. This is contrary to the findings by Giiti et al (2010) at BMC who found a highly significant association between the duration of hospital stay and HIV serostatus. We could not establish the reason for the lack of association between the duration of hospital stay and HIV serostatus. This calls for a need to research on this observation. Our mortality rate for HIV positive was significantly higher than that of HIV negative patients. In keeping with other studies (Moody et al., 2010; Lapadula et al., 2013; Feng et al., 2015) the mortality rate for HIV positive patients with stigmata of AIDS and those with CD4 count $<200$ cells $/ \mu \mathrm{L}$ was significantly higher than in patients without stigmata of AIDS and those with CD4 count $\geq 200$ cells $/ \mu \mathrm{L}$. This observation may be explained by the fact that HIV infection causes impaired immunity predisposing them to increased risk of death.

The potential limitation of this study is the limited follow-up time and difficult to diagnose HIV infection in its early stage. Also, the management of our patients from thee different levels of hospitals; tertiary, regional and district taking into consideration the difference in facilities present at these levels such as diagnostics, specialists and nurses may have affected the clinical outcomes However, despite these limitations, the study has provided local data that can guide health care providers in the treatment of these patients.

In conclusion, this study has shown that HIV infection is prevalent among patients with surgical acute abdomen in north-western Tanzania. Factors affecting clinical outcomes of these patients need to be addressed in order to deliver optimal care for these patients and improve their treatment outcomes.

\section{Acknowledgments}

The authors acknowledge all those who participated in the preparation of this manuscript and those who were involved in the care of our study patients

\section{References}

Aboolian, A., Ricci, M., Shapiro, K, Connors, A. \& LaRaja, R. D. (1999) Surgical treatment of HIVrelated immune thrombocytopenia. International Surgery 84: 81-85. 
Amira, C.O. \& Awobusuyi, J.O. (2014) Needle-stick injury among health care workers in hemodialysis units in Nigeria: a multi-center study. International Journal of Occupational Medicine and Environmental Health 5: 1-8.

Amita, G., Shuchi, A., Jaygow, Anandini, K., Anita, B., Shreepad, M.B., Nkhil, G., Robert, C.B. \& Arjun, C.K. (2008) High risk for occupational exposure to HIV and utilization of post-exposure prophylaxis in a teaching hospital in Pune, India. BMC Infectious Disease 8: 142.

Bhuiya, F.A., Pitts, S.R., McCaig, L.F. (2006) Emergency department visits for chest pain and abdominal pain: United States, 1999-2008. NCHS Data Brief 43: 1-8.

Cacala, S.R., Mafana, E., Thompson, S.R., Smith, A. (2006) Prevalence of HIV status and CD4 counts in a surgical cohort: their relationship to clinical outcome. Annals of the Royal College of Surgeons of England 88: 46-51.

CDC (2006) Revised recommendations for HIV testing of adults, adolescents, and pregnant women in health-care settings. MMWR 55(RR-14).

Chalya, G., Mbunda, F. \& Chalya, P.L. (2016) Knowledge, practice and factors associated with poor compliance with universal precautions among healthcare workers at Bugando Medical Centre, Mwanza, Tanzania. Tanzania Journal of Health Research 18: 3

Chalya, P.L., Mchembe, M.D., Mshana, S.E., Rambau, P.F., Jaka, H. \& Mabula, J.B. (2013a) Clinicopathological profile and surgical treatment of abdominal tuberculosis: a single centre experience in northwestern Tanzania. BMC Infectious Diseases 13: 270.

Chalya, P.L., Mchembe, M.D., Mshana, S.E., Rambau, P., Jaka, H. \& Mabula, J.B. (2013b) Tuberculous bowel obstruction at a university teaching hospital in Northwestern Tanzania: a surgical experience with 118 cases. World Journal of Emergency Surgery 8: 12.

Chalya, P.L., Mabula, J.B., Koy, M., Mchembe, M.D., Jaka, H.M., Kabangila, R., Chandika, A.B. \& Gilyoma, J.M. (2011) Clinical profile and outcome of surgical treatment of perforated peptic ulcers in Northwestern Tanzania: A tertiary hospital experience. World Journal of Emergency Surgery 6: 31.

Chalya, P.L, Mabula, J.B., Koy, M., Kataraihya, J.B., Jaka, H., Mshana, S.E., Mirambo, M., Mchembe, M.D., Giiti, G. \& Gilyoma, J.M. (2012) Typhoid intestinal perforations at a University teaching hospital in Northwestern Tanzania: A surgical experience of 104 cases in a resource-limited setting. World Journal of Emergency Surgery 7, 4.

Davis, P.A., Corless, D.J. \& Aspinilla, R. (2001) Effect of CD4 (+) and CD8 (+) cells depression on wound healing. British Journal of Surgery 88: 298-304.

Dua, R.S. \& McWinslet, S. (2007) Impact of HIV and AIDS in Surgical practice. . Annals of the Royal College of Surgeons of England 89: 354-358.

Feng, T., Feng, X., Jiang, C, Huang, C. \& Liu, B. (2015) Sepsis risk factors associated with HIV-1 patients undergoing surgery. Emerging Microbes \& Infections 4: e59.

Gazard, B.G., Wastell, C., Davis, P.A. \& Corless, D.J. (1999) Increase risk of wound complications and poor healing following laparotomy in HIV seropositive and AIDS patients. Digestive Surgery 16: 60-67.

Giiti, G.C., Mazigo, H.D., Heukelbach, J. \& Mahalu, W. (2010) HIV, appendectomy and postoperative complications at a reference hospital in Northwest Tanzania: cross-sectional study. AIDS Research and Therapy 7: 47.

Green, S., Kong, V.Y., Odendaal, J., Sartorius, B., Clarke, D.L., Brysiewicz, P., Bruce, J.L., Laing, G.L. \& Bekker, W. (2017) The effect of HIV status on clinical outcomes of surgical sepsis in KwaZulu-Natal Province, South Africa. South African Medical Journal 107: 702-705.

Hastings, R.S., Powers, R.D. (2011) Abdominal pain in the ED: A 35 year retrospective. American Journal of Emergency Medicine 29: 711-716.

Johnston, L.G., Sabin, M.L., Prybylski, D., Sabin, K., McFarland, W., Baral, S., Kim, A.A. \& Raymond, H.F. (2016) Policy and practice: the importance of assessing self-reported HIV status in bio-behavioural surveys. Bulletin of the World Health Organization 94: 605-612. 
Khojah, I., Li, S., Luo, Q., Davis. G., Galarraga, J.E., Granovsky, M., Litvak, O., Davis, S., Shesser, R., Pines, J.M. (2017) The relative contribution of provider and ED-level factors to variation among the top 15 reasons for ED admission. American Journal of Emergency Medicine 35: 1291-1297.

Lapadula, G., Cozzi-Lepri, A., Marchetti, G., Antinori, A., Chiodera, A., Nicastri, E., Parruti, G., Galli, M., Gori, A. \& Monforte, A. (2013) Risk of clinical progression among patients with immunological nonresponse despite virological suppression after combination antiretroviral treatment. AIDS 27: 769-779.

Lyamuya, E.F., Aboud, S., Urassa, W.K., Sufi, J., Mbwana, J., Ndugulile, F. \& Massambu, C. (2009) Evaluation of simple rapid HIV assays and development of national rapid HIV test algorithms in Dar es Salaam, Tanzania. BMC Infectious Disease 9: 19.

Makanga, M.R.K., Nzayisegna, A. \& Kakande, I. (2006) HIV/AIDS among Surgical Patients in Butare University Teaching Hospital. East and Central Africa Journal of Surgery 12: 123-125.

Mabula, J.B., Chalya, P.L., Mchembe, M.D., Kihunrwa, A., Massinde, A., Chandika, A.B. \& Gilyoma, J.M. (2012) Bowel perforation secondary to illegally induced abortion: a tertiary hospital experience in Tanzania. World Journal of Emergency Surgery 7: 29.

Mawalla, B., Mshana, S.E., Chalya, P.L., Imirzalioglu, C. \&, Mahalu, W. (2011) Predictors of surgical site infections among patients undergoing major surgery at Bugando Medical Centre in Northwestern Tanzania. BMC Surgery 11: 21.

Mabewa, A., Seni, J., Chalya, P,L., Mshana, S.E. \& Gilyoma, J.M. (2015) Etiology, treatment outcome and prognostic factors among patients with secondary peritonitis at Bugando Medical Centre, Mwanza, Tanzania. World Journal of Emergency Surgery 10: 47.

Mkony, C., Kwesigabo, G., Lyamuya, E. \& Mhalu, F. (2003) Prevalence and clinical presentation of HIV infection among newly hospitalized surgical patients at Muhimbili National Hospital, Dar es Salaam, Tanzania. East and Central Africa Journal of Surgery 80: 640-645.

Morales, M.J., Zúñiga, Z.M., Silva, G.M., Cortés, M.L., Cortés, V.S.R., Jadan, J.C.B. \& Valdez, O.V. (2017) Evaluation and management of abdominal pain in patients with Human Immunodeficiency Virus: a review of literature. SM Journal of Public Health \& Epidemiology 3: 1042.

Moody, F., Deneve, J.L., Grant, W. (2010) CD4 count is predictive of outcome in HIV-positive patients undergoing abdominal operations. American Journal of Surgery 200: 699-700.

Owotade, F.J., Ogunbodede, E.O. \& Sowande, O.A. (2003) HIV? AIDS pandemic and surgical practice in a Nigerian teaching hospital. Tropical Doctor 33: 228-231

Tan, A.X., Kapiga, S., Khoshnood, K. \& Bruce, R.D. (2015) Epidemiology of drug use and HIV-related risk behaviours among people who inject drugs in Mwanza, Tanzania. PLoS ONE 10: e0145578. 